02

\title{
Взаимосвязь между молекулярной динамикой полистирола и его модификаций и параметрами терагерцового поглощения в ИК спектрах
}

\author{
(C) B.A. РЫжов
}

ФТИ им. А.Ф. Иофрфе РАН, 194021 Санкт-Петербург, Россия

e-mail: v.ryzhov@mail.ioffe.ru

Поступила в редакцию 22.10.2020 г.

В окончательной редакции 22.10.2020 г.

Принята к публикации 19.11.2020 г.

Измерены и проанализированы ИК спектры полистирола (ПС) и его модификаций, полученных замещениями атомов водорода в бензольном кольце метильными группами и/или атомами хлора и брома. Все эти спектры имеют аномально широкую асимметричную полосу поглощения с положением максимума в терагерцовом диапазоне от 40 до $80 \mathrm{~cm}^{-1}$ в зависимости от природы заместителя. Она может быть отнесена к поглощению, связанному с либрацией (вращательными колебаниями) фенольных колец мономерных звеньев, т. е. к поглощению по механизму Поли. Взаимосвязь между спектральными параметрами этого поглощения и молекулярными характеристиками изученных полимеров позволяет непосредственно анализировать роль молекулярной структуры и межмолекулярных сил в динамике их макромолекул. Обнаружено, что высоты потенциальных барьеров либрации мономерных единиц ПС и его модификаций, оцененные на основе анализа поглощения Поли, близки к энергиям активации низкотемпературной $\delta$-релаксации в этих стеклообразных полимерах. Сравнение барьеров либрации мономерных единиц макромолекул ПС и его модификаций с активационными барьерами локальной сегментальной подвижности в тех же ПС подтверждает, что $\beta$-процесс как элементарный акт сегментальной динамики обусловлен коррелированным либрационным движением участка цепи, статистически независящего от соседних участков. В этом смысле универсальный $\delta$-процесс является высокочастотным предшественником $\beta$-процесса в полимерах, подобных ПС.

Ключевые слова: молекулярная динамика, $\beta$-релаксация, стеклование, полистирол и его модификации, ИК спектры.

DOI: $10.21883 /$ OS.2021.03.50655.264-20

\section{Введение}

Влияние особенностей молекулярного строения и взаимодействий полимерных молекул на их локальную и сегментальную динамику по-прежнему остается в кругу теоретических и экспериментальных исследований $[1,2]$. В последние годы существенные успехи в этом направлении были достигнуты при совместном использовании высокочастотной диэлектрической и низкочастотной колебательной (ИК и Раман) спектроскопии благодаря их уникальной способности непосредственно анализировать особенности неупорядоченной конденсированной среды, определяемые как индивидуальными, так и коллективными свойствами молекул и природой межмолекулярных сил $[3,4]$.

Использование терагерцового диапазона частот в таких исследованиях представляет особый интерес, поскольку здесь спектр релаксационного типа и резонансный спектр имеют тенденцию сосуществовать, и, таким образом, низкочастотные колебательные спектры являются прямым источником информации о молекулярной подвижности, предшествующей релаксационной динамике в стеклообразных полимерах. Речь идет о проявлении в интервале $10-130 \mathrm{~cm}^{-1}$ ИК спектров жидкостей и неупорядоченных материалов, включая полимеры, аномально широкой полосы поглощения, обусловленной малоугловыми вращательными колебаниями молекул [5-7]. Такие малоугловые вращательные колебания (либрации) фенольных колец в мономерных звеньях полистирола (ПС), ответственные за $\delta$-релаксацию в этом полимере, проявляются в ИК спектре ПС широкой полосой поглощения с максимумом при $80 \mathrm{~cm}^{-1}[8,9]$.

Либрационное поглощение (также называемое поглощением по механизму Поли) в ИК спектрах полимеров изучалось как теоретически, так и экспериментально. Было показано, что его интенсивность пропорциональна величине $\mu^{2} / I$, где $\mu$ и $I$ - дипольный момент и момент инерции боковой группы (фенольного кольца) полимера соответственно. Положение максимума этого поглощения пропорционально $(V / 2 I)^{1 / 2}$, где $V$ - высота потенциального барьера для либрации, сопоставимого по величине с энергией активации $\delta$-перехода в ПС $[8,10]$. Найденная связь между спектральными параметрами поглощения Поли с молекулярными характеристиками полимеров позволяет проанализировать роль молекулярной структуры и межмолекулярных сил в динамике макромолекул. 
В настоящей работе найденные корреляции применялись для определения потенциальных барьеров крутильных колебаний в макромолекулах ПС и его производных, отличающихся строением бензольного кольца. Проведение исследований на подобной серии полимерных объектов, когда задаваемым и контролируемым образом варьируются их молекулярные параметры, позволяет выявить роль электронной структуры и стерического фактора в таких элементарных актах сегментального движения, как крутильные колебания мономерных звеньев, подготавливающие релаксационную подвижность макромолекул.

Полученные результаты сравнивались с данными других методик, и, в частности, с оценками и выводами работы [11], выполненной на таких же объектах методом ДСК и УФ спектроскопии.

\section{Методика}

Объектами исследования были ПС и его модификации, синтезированные с помощью сложной многоступенчатой процедуры; это привело к созданию набора полимеров с контролируемым изменением структуры бензольного кольца. Структуру ароматического кольца изменяли путем замещения метильными группами $\left(\mathrm{CH}_{3}\right)$ и/или атомами хлора $(\mathrm{Cl})$ и брома $(\mathrm{Br})$ атомов водорода в разных положениях на кольце с различным числом замещений и различной комбинацией заместителей [12]. Перечень образцов и некоторые их теплофизические и энергетические характеристики представлены в табл. 1. Молекулярные массы полимеров лежали в диапазоне $(1-2) \cdot 10^{5}$. Молекулярные массы и ван-дер-ваальсовы объемы их мономерных звеньев также приведены в табл. 1.

ИК спектры регистрировали на однолучевых вакуумных спектрометрах с дифракционными решетками от 10 до $150 \mathrm{~cm}^{-1}$ на приборе, разработанном в Петербургском государственном университете и модернизированном в ФТИ с помощью приемника ОАП-7 и новой системы фильтрации, и от 150 до $450 \mathrm{~cm}^{-1}$ на спектрометре „Нitachi“ FIS-21 (Япония). Разрешение при отношении сигнала к шуму порядка 100 составляло $1-2 \mathrm{~cm}^{-1}$. Точность определения частоты максимума полосы составляла $0.5-1 \mathrm{~cm}^{-1}$.

Коэффициент поглощения $k(v)=\ln \left(J_{0} / J\right) /\left(t-t_{0}\right)$, где $J_{0}$ и $J$ - пропускание образцов толщиной $t_{0}$ и $t$, измерялся с погрешностью от 5 до 10\%. Все спектры были измерены для образцов, полученных в виде пленок толщиной от 200 до $300 \mu \mathrm{m}$, измерения проводились при комнатной температуре.

\section{Результаты и их обсуждение}

На рис. 1, $a-l$ показаны ИК спектры ПС и его 11 модификаций в области $20-450 \mathrm{~cm}^{-1}$. Поглощение на этих частотах в спектрах замещенных бензолов, стиролов и ПС, как установлено $[17,18]$, обусловлено, главным образом, деформационными колебаниями бензольных колец (моды 9b, 10b и 16a), смешанными в полимере с деформационными колебаниями основной цепи, а также крутильными колебаниями мономерных единиц и $\mathrm{CH}_{3}$-группы, если таковая имеется.

Предложенное в работах $[15,16]$ отнесение низкочастотных полос, наблюдаемых в ИК спектре ПС (рис. 1,a), может быть представлено следующим образом: интенсивная полоса при $\sim 405 \mathrm{~cm}^{-1}-$ внеплоскостное деформационное колебание связи $\mathrm{C}-\mathrm{C}$ ароматического кольца (мода 16а); слабые полосы при 325 и $295 \mathrm{~cm}^{-1}$ обусловлены деформационными колебаниями $\mathrm{C}-\mathrm{C}-\mathrm{C}-$ и $\mathrm{C}-\mathrm{C}-\mathrm{H}-г$ рупп основной цепи. Наиболее интенсивная полоса в ИК спектре ПС с максимумом при $218 \mathrm{~cm}^{-1}$ имеет сложный контур, состоящий, по меньшей мере, из двух компонент при 218 и $245 \mathrm{~cm}^{-1}$. Соответствующая пара полос наблюдалась для модельных соединений ПС [17], и одна компонента дублета (при $218 \mathrm{~cm}^{-1}$ ) была отнесена к моде 9b, а другая (при $\left.245 \mathrm{~cm}^{-1}\right)$ к скелетным колебаниями основной цепи. Мода $10 \mathrm{~b}$ внеплоскостных колебаний связей $\mathrm{C}-\mathrm{H}$ ароматического кольца, предсказанная расчетом для замещенных ПС в диапазоне от 150 до $200 \mathrm{~cm}^{-1}$, в ИК спектре ПС отдельной полосой не проявляется. И, наконец, низкочастотная аномально широкая полоса поглощения в ИК спектре ПС с максимумом примерно при $80 \mathrm{~cm}^{-1}$ (рис. 2, $a$ ) в основном обусловлена крутильными колебаниями (либрацией) ароматического кольца, т. е. относится к поглощению по механизму Поли $[18,19]$. Вышеприведенное отнесение наблюдаемых полос в ИК спектре ПС позволяет достаточно уверенно идентифицировать основные полосы поглощения в ИК спектрах модифицированных ПС.

Из представленных на рис. 1 и 2 спектров видно, что при замещении атомов водорода в бензольном кольце атомами $\mathrm{Cl}, \mathrm{Br}$ или $\mathrm{CH}_{3}$-группами наиболее существенно изменяется контур ИК спектра на частотах проявления поглощения по механизму Поли в области $10-120 \mathrm{~cm}^{-1}$, а также в интервале $150-350 \mathrm{~cm}^{-1}$, где находятся полосы колебаний $9 \mathrm{~b}$ и $10 \mathrm{~b}$. Заметим, что привлечение последних для качественного анализа и при установлении различных корреляций следует все же проводить с большой осторожностью. Подобное замечание относится и к информативности полосы колебания 16а, проявляющегося вблизи $405 \mathrm{~cm}^{-1}$ в ИК спектрах ПС и всех его модификаций, представленных здесь, независимо от числа, типа и положения заместителя в бензольном кольце.

Наиболее перспективным для анализа и установления взаимосвязи параметров ИК спектров с молекулярными характеристиками и динамикой исследуемых полимеров является использование спектральных параметров поглощения в диапазоне ниже $150 \mathrm{~cm}^{-1}$, поскольку теоретические предсказания исключают участие внутренних мод на этих частотах [20]. В настоящее время хорошо установлено, что основной составляющей ИК спектров 
Таблица 1. Молекулярные характеристики ПС и его модификаций

\begin{tabular}{|c|c|c|c|c|c|c|c|c|}
\hline $\begin{array}{c}\text { № } \\
\text { поли- } \\
\text { мера }\end{array}$ & Полимер & $\begin{array}{c}\text { Van der Waals } \\
\text { объем } V, \\
10^{-} 24 \mathrm{~cm}^{3}\end{array}$ & $\begin{array}{c}\text { Молекулярная } \\
\text { масса } M, \\
10^{-27} \mathrm{~kg}\end{array}$ & $\begin{array}{c}\text { Радиус } R, \\
10^{-10} \mathrm{~m} \\
\text { боковой } \\
\text { группы }\end{array}$ & $\begin{array}{c}\text { Момент } \\
\text { инерции } \\
I_{\mu}=0.4 M R^{2}, \\
10^{-47} \mathrm{~kg} \cdot \mathrm{m}^{2}\end{array}$ & $\begin{array}{c}\text { Дипольный } \\
\text { момент } \mu, \\
3.33 \cdot 10^{-30} \mathrm{C} \mathrm{m}\end{array}$ & $\begin{array}{c}\text { Энергия } \\
\text { когезии } \\
E_{\mathrm{coh}}, \\
10^{-19} \mathrm{~J}\end{array}$ & $\begin{array}{c}\mu^{2} / I_{\mu}, \\
10^{-16} \\
\mathrm{C}^{2} \mathrm{~kg}^{-1}\end{array}$ \\
\hline 1 & Полистирол & 85 & 134 & 2.8 & 420 & 0.18 & 0.46 & 0.85 \\
\hline 2 & $\begin{array}{l}\text { Поли-2-метил } \\
\text {-стирол }\end{array}$ & 104 & 160 & 2.96 & 560 & 0.39 & 0.58 & 3.02 \\
\hline 3 & $\begin{array}{l}\text { Поли-4- } \\
\text { метил-стирол }\end{array}$ & 103 & 160 & 2.95 & 560 & 0.36 & 0.56 & 2.56 \\
\hline 4 & $\begin{array}{l}\text { Поли-2,4- } \\
\text { диметил-стирол }\end{array}$ & 115 & 182 & 3.07 & 690 & 0.37 & 0.66 & 2.22 \\
\hline 5 & $\begin{array}{l}\text { Поли-2,5- } \\
\text { диметил-стирол }\end{array}$ & 115 & 182 & 3.07 & 690 & 0.32 & 0.58 & 1.65 \\
\hline 6 & $\begin{array}{l}\text { Поли-4- } \\
\text { хлор-стирол }\end{array}$ & 112 & 195 & 3.03 & 720 & 1.65 & 0.57 & 43 \\
\hline 7 & $\begin{array}{l}\text { Поли-4-хлор } \\
\text {-3-метил-стирол }\end{array}$ & 117 & 216 & 3.08 & 820 & 1.82 & 0.58 & 44 \\
\hline 8 & $\begin{array}{l}\text { Поли-2-хлор-3,4- } \\
\text { диметил-стирол }\end{array}$ & 135 & 240 & 3.24 & 1000 & 1.70 & 0.69 & 31.5 \\
\hline 9 & $\begin{array}{l}\text { Поли-2-хлор-3,5- } \\
\text { диметил-стирол }\end{array}$ & 135 & 240 & 3.24 & 1000 & 1.50 & 0.77 & 24.8 \\
\hline 10 & $\begin{array}{l}\text { Поли-2,4,5-три } \\
\text {-метил-стирол }\end{array}$ & 126 & 306 & 3.16 & 1230 & 1.70 & 0.67 & 25.7 \\
\hline 11 & $\begin{array}{l}\text { Поли-4-бром-2, } \\
\text { 5-диметилстирол }\end{array}$ & 138 & 314 & 3.26 & 1340 & 1.65 & 0.83 & 22.5 \\
\hline 12 & $\begin{array}{l}\text { Поли-2,3-дихлор-4,5 } \\
\text {-диметил-стирол }\end{array}$ & 150 & 300 & 3.35 & 1350 & 2.40 & 0.8 & 47 \\
\hline
\end{tabular}

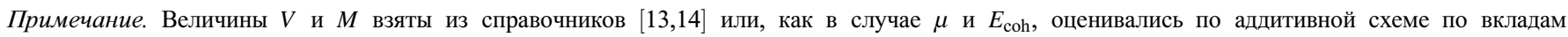
функциональных групп.

полимеров в диапазоне ниже $150 \mathrm{~cm}^{-1}$ является поглощение по механизму Поли (Poley-type absorption). Это аномально широкое поглощение в диапазоне частот от 10 до $130 \mathrm{~cm}^{-1}$ не уникально для полимеров, оно также характерно для жидкостей и многих неупорядоченных твердых тел, например для стекол [19,21].

Появление поглощения в области ниже $150 \mathrm{~cm}^{-1}$ может быть описано моделью затухающих вращательных колебаний (либраций) полярных молекул. Одна из наиболее простых моделей молекулярного движения, применяемая в настоящее время для анализа этого поглощения, - модель ограниченных ротаторов или либрации молекулы в потенциальной яме Бро-Дармона [10,22]. Согласно этой модели, либрационное движение полярной молекулы с моментом инерции I совершается в пределах образованной ее ближайшим окружением потенциальной ямы, имеющей форму $U(\varphi)=V_{0} \sin ^{2} \pi \varphi / 2 \xi$, где $V_{0}$ - глубина ямы, $\xi$ - ее полуугловая апертура (ширина на высоте, равной половине полного барьера) и $\varphi-$ угол либрации (амплитуда ограниченных качаний). Движение совершается с круговой частотой $\omega_{0}=2 \pi c \nu_{\text {libr }}=\pi \xi^{-1}\left(V_{0} / 2 I\right)^{1 / 2}$. Интегральная интенсивность поглощения определяется выражением

$$
A=\int k(v) d v=\pi / 3 c^{2} \sum \mu_{z}^{2}\left(1 / I_{x}+1 / I_{y}\right),
$$

где $k(v)$ - коэффициент поглощения, $\mu_{z}$ - дипольный момент вдоль молекулярной оси, $I_{x}$ и $I_{y}-$ моменты инерции, перпендикулярные этой оси. Это либрационное Poley-type absorption в ИК спектрах полимеров предоставляет информацию, принципиально отличающуюся от получаемой из спектров средней ИК области, поскольку оно характеризуется большей ролью межмолекулярной динамики.

Полосы Poley-type absorption в ИК спектрах исследованных производных ПС приведены в увеличенном масштабе на рис. 2. Как видно, интенсивность и положение этих широких (с полушириной $\Delta v=30-50 \mathrm{~cm}^{-1}$ ) полос существенно изменяется при вариации структуры бензольного кольца замещениями. В зависимости от типа заместителя, их количества и положение в бензольном кольце интенсивность этого поглощения $\left(k_{\max }\right)$ может увеличиться в несколько раз, а положение его максимума $\left(v_{\max }\right)$ сместиться на $30-40 \mathrm{~cm}^{-1}$ по сравнению с ИК спектром ПС (табл. 2).

Взаимосвязь значений $k_{\max }$ и $v_{\max }$ с такими молекулярными характеристиками исследованных полимеров, как эффективный дипольный момент боковой группы $(\mu)$, ее геометрия (момент инерции $I$ ) и энергия когезии $E_{\text {coh }}$, характеризующая межмолекулярное взаимодействие в полимерах [13], иллюстрируют кривые на рис. $3, a, b$. 

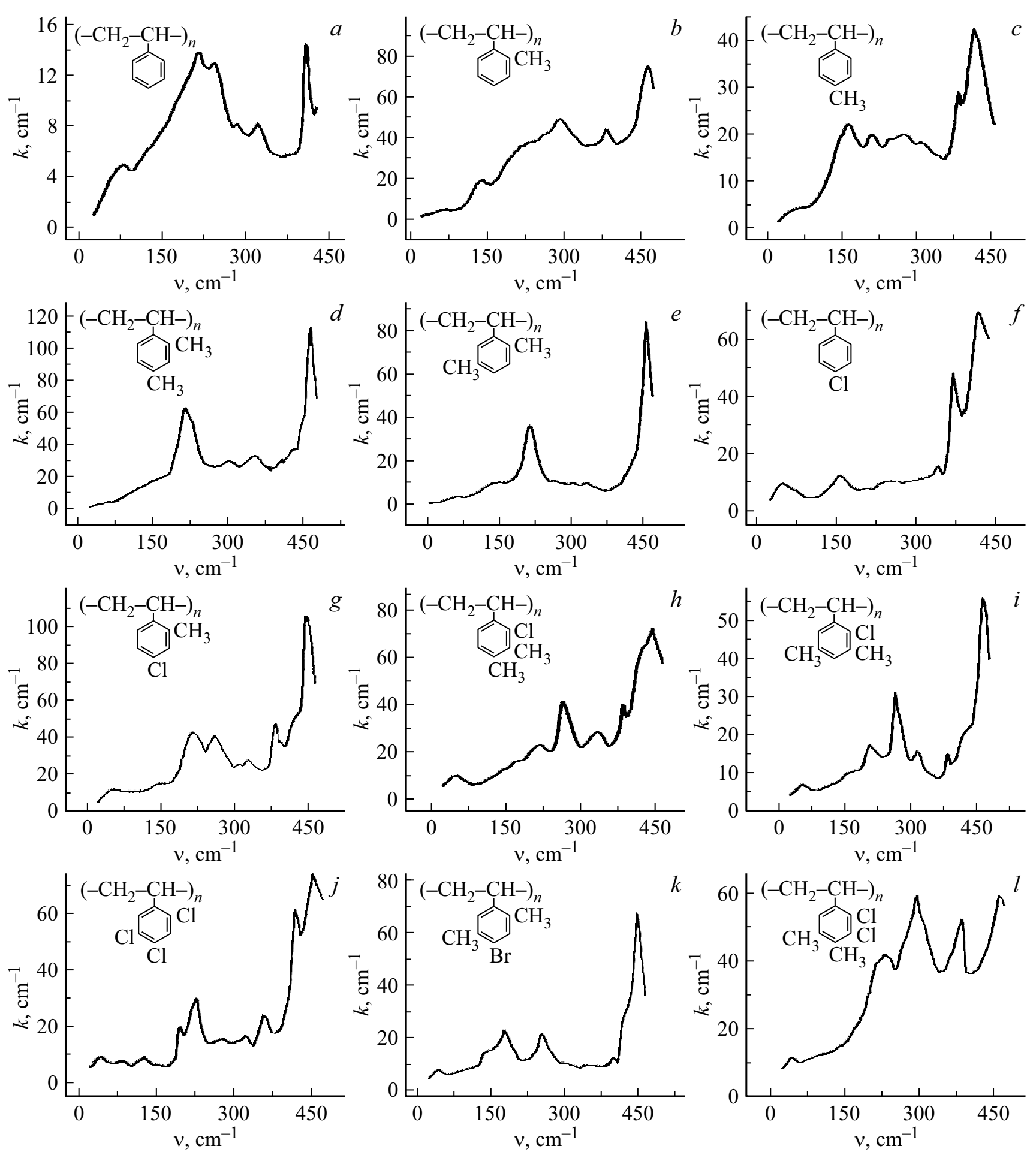

Рис. 1. ИК спектры ПС и его модификаций. ПС $(a)$; поли-2-метилстирол $(b)$; поли-4-метилстирол $(c)$; поли-2,4-диметилстирол $(d)$; поли-2,5-диметилстирол $(e)$; поли-4-хлоростирол $(f)$; поли-4-хлор-3-метилстирол $(g)$; поли-2-хлор-3,4-диметил-стирол $(h)$; поли-2-хлор-3,5-диметилстирол $(i)$; поли-2,4,5-три хлор-стирол $(j)$; поли-4-бром-2,5-диметилстирол $(k)$; Поли-2,3-дихлор-4,5диметилстирол $(l)$.

Рисунок 3, $a$ показывает, что для исследованных полимеров имеет место пропорциональная зависимость между $k_{\max }$ и величиной $\mu^{2} / I$, непосредственно свидетельствующая о либрационной природе этого поглощения.
В соответствии с этим отнесением находится и иллюстрированная рис. $3, b$ пропорциональность между $v_{/ \max }$ и величиной $\left(E_{\mathrm{coh}} / 2 I\right)^{1 / 2}$, отражающая зависимость потенциального барьера для ограниченных крутильных 

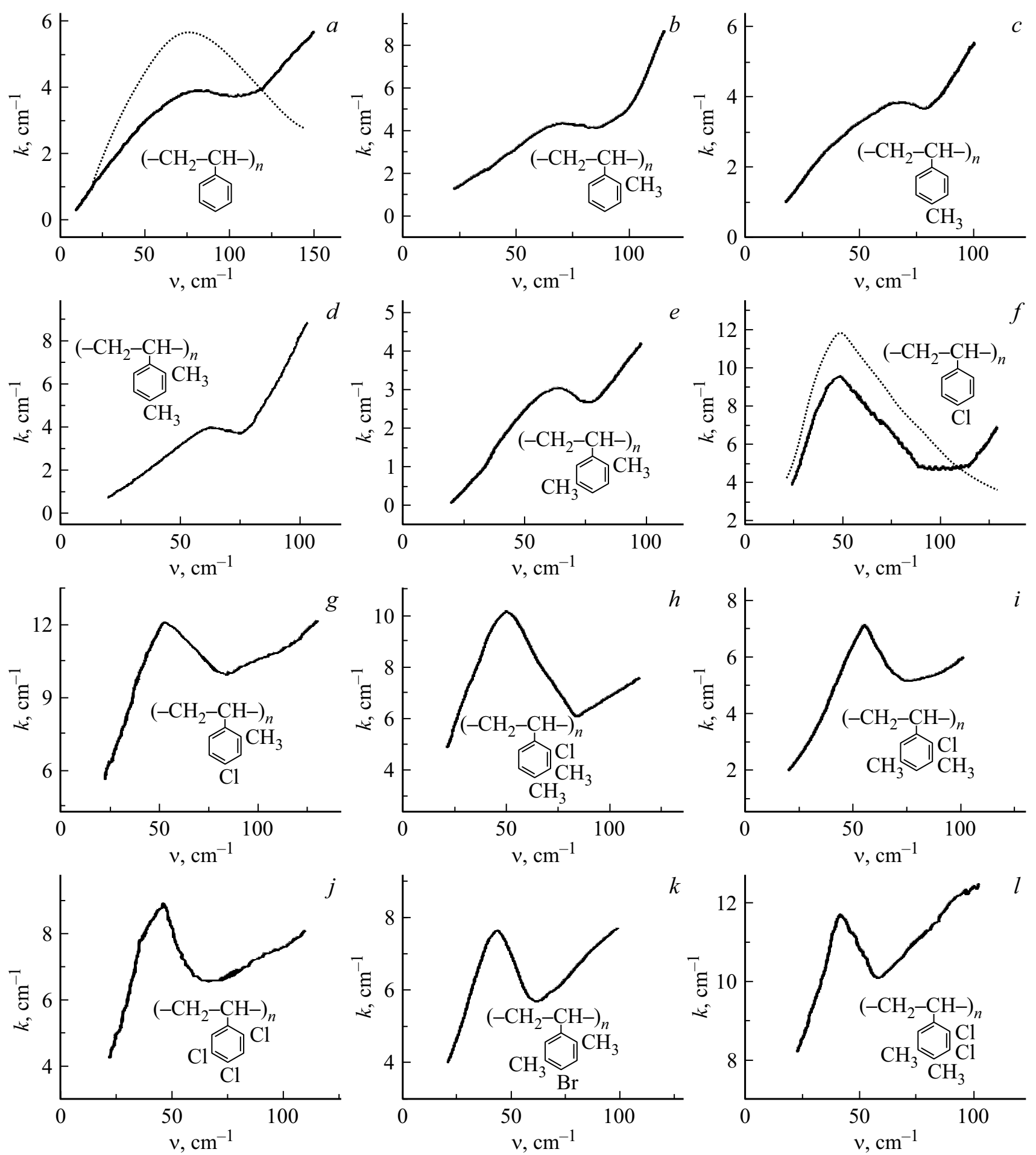

Рис. 2. Полоса поглощения Поли в ИК спектрах ПС $(a)$ и его модификаций $(b-l)$. Обозначения те же, что на рис. 1 . Пунктирными линиями на рис. $2, a$ и $2, f$ показаны спектры $\mathrm{C}_{6} \mathrm{H}_{6}$ и $\mathrm{C}_{6} \mathrm{H}_{5} \mathrm{Cl}$ соответственно.

колебаний (либрации) от уровня межмолекулярных взаимодействий в исследованных полимерах.

Обнаруженные корреляции позволяют, используя модель Бро-Дармона, оценить потенциальные барьеры для либрации боковых групп в макромолекулах ПС и его модификациях. Согласно такому подходу, при малых уг- лах крутильных колебаний либрационная частота $\left(v_{\text {libr }}\right)$ и потенциальный барьер для либрации $\left(Q_{\text {libr }}\right)$ связаны соотношением

$$
\nu_{\text {libr }}=(c \pi \varphi)^{-1}\left(Q_{\text {libr }} / 2 I\right)^{1 / 2} .
$$


Таблица 2. Взаимосвязь параметров Poley-type absorption и параметров локальной сегментальной подвижности в ПС и его модификациях. Номера полимеров соответствуют номерам в табл. 1

\begin{tabular}{|c|c|c|c|c|c|c|c|c|c|c|}
\hline $\begin{array}{l}\text { № } \\
\text { по- } \\
\text { ли- } \\
\text { ме- } \\
\text { pa }\end{array}$ & $\begin{array}{c}\text { Положение } \\
\text { максимума } \\
v_{\max }, \mathrm{cm}^{-1}\end{array}$ & $\begin{array}{c}\text { Интен- } \\
\text { сивность } \\
k_{\max } \\
\mathrm{cm}^{-1}\end{array}$ & $\begin{array}{c}\text { Барьер } \\
Q_{\text {libr }}=7.1 \times \\
10^{20} I_{\text {libr }}\left(v_{\max }\right)^{2}, \\
10^{-19} \mathrm{~J}\end{array}$ & $\begin{array}{c}\text { Энергия } \\
\text { когезии } \\
E_{\text {coh }}, \\
10^{-19} \mathrm{~J}\end{array}$ & $\begin{array}{c}\left(E_{\mathrm{coh}} / 2 I\right)^{1 / 2}, \\
10^{10} \\
(\mathrm{~kJ} / \\
\left./ \mathrm{kgm}^{2}\right)^{1 / 2}\end{array}$ & $E_{\mathrm{coh}} / Q_{\mathrm{libr}}$ & $\begin{array}{c}\text { Стати- } \\
\text { стиче- } \\
\text { ский } \\
\text { сегмент } \\
S \\
\text { (число } \\
\text { моно- } \\
\text { меров) }\end{array}$ & $\begin{array}{c}T_{g}, \mathrm{~K}, \\
\text { по дан- } \\
\text { ным } \\
\text { DSC } \\
\text { из [13] }\end{array}$ & $\begin{array}{c}U_{\alpha}, \cdot 10^{-19} \mathrm{~J}, \\
\text { по дан- } \\
\text { ным } \\
\text { DSC } \\
\text { из }[13]\end{array}$ & $\begin{array}{c}U_{\beta}=Q_{\mathrm{libr}} S \\
10^{-19} \mathrm{~J}\end{array}$ \\
\hline 1 & 72.0 & 1.4 & 0.152 & 0.60 & 7.4 & 3.0 & 8.0 & 372 & 1.64 & 1.3 \\
\hline 2 & 63.0 & 1.2 & 0.160 & 0.73 & 7.2 & 3.5 & 8.0 & 396 & 1.74 & 1.3 \\
\hline 3 & 61.0 & 1.9 & 0.150 & 0.71 & 7.1 & 3.7 & 8.0 & 386 & 1.70 & 1.2 \\
\hline 4 & 60.0 & 1.8 & 0.175 & 0.80 & 6.9 & 3.7 & 8.0 & 409 & 1.80 & 1.4 \\
\hline 5 & 58.0 & 1.1 & 0.170 & 0.72 & 6.5 & 3.4 & 8.0 & 392 & 1.73 & 1.4 \\
\hline 6 & 50.5 & 5.5 & 0.130 & 0.73 & 6.3 & 4.3 & 11.0 & 405 & 1.76 & 1.4 \\
\hline 7 & 51.0 & 6.0 & 0.150 & 0.72 & 5.9 & 3.8 & 8.0 & 394 & 1.73 & 1.2 \\
\hline 8 & 49.4 & 5.1 & 0.177 & 0.83 & 5.9 & 3.8 & 9.0 & 410 & 1.80 & 1.6 \\
\hline 9 & 55.3 & 4.2 & 0.220 & 0.91 & 6.2 & 3.5 & 8.0 & 420 & 1.85 & 1.8 \\
\hline 10 & 45.1 & 4.6 & 0.187 & 0.84 & 5.2 & 3.6 & 9.0 & 410 & 1.80 & 1.7 \\
\hline 11 & 43.8 & 3.7 & 0.184 & 1.00 & 5.6 & 4.5 & 10.0 & 426 & 1.87 & 1.8 \\
\hline 12 & 41.5 & 5.6 & 0.166 & 0.97 & 5.4 & 4.8 & 11.0 & 422 & 1.86 & 1.8 \\
\hline
\end{tabular}
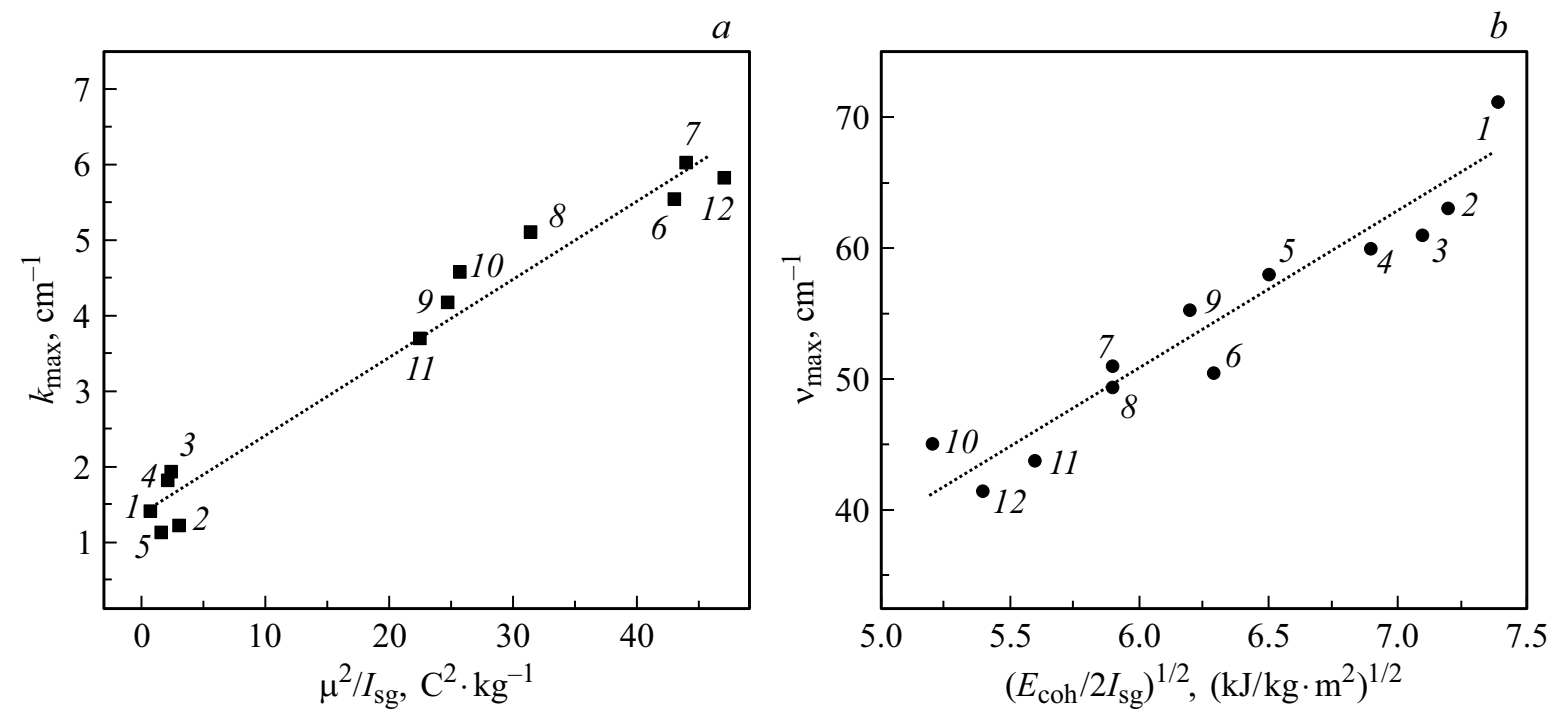

Рис. 3. Зависимость интенсивности полосы „Роley-type absorption“ $(a)$ и ее частоты в максимуме $(b)$ от величин молекулярных параметров полимеров. Нумерация точек та же, что в табл. 2.

Отметим, что модель Бро-Дармона для полярных жидкостей дает хорошее согласие с экспериментом, а потенциальные барьеры либрации, рассчитанные с использованием этой модели, близки к тем, которые получены другими способами [23]. Расчет $Q_{\text {libr }}$ производился в двух вариантах: в предположении о либрации боковой группы и при рассмотрении в качестве либратора мономерного звена как целого. При расчете условно принимался шаровой ван-дер-ваальсовый объем либратора, и момент инерции определялся по формуле $I=0.4 M R^{2}$, где $M$ - молекулярный вес и $R$-- радиус боковой группы. Угол либрации $\varphi$ принимался равным $12.5^{\circ}$ на основе теоретических моделей и расчетов, которые указывают на наличие в макромолекулах ограниченных крутильных колебаний атомных групп с амплитудой от $10^{\circ}$ до $15^{\circ}$ [20].

Результаты расчета барьеров либрации боковых групп в макромолекулах ПС и его модификациях $Q_{\text {libr }}$ приведены в табл. 2 (столбец 4), где они сравниваются с взятыми из [13] энергиями когезии $E_{\text {coh }}$ (столбец 5) боковых групп этих же полимеров. Примечательная близость $Q_{\mathrm{libr}}$ к $E_{\mathrm{coh}} / 3$; она указывает на межмоле- 
кулярную природу барьера либрации. Вклад в $Q_{\text {libr }}$ от барьера внутреннего вращения $Q_{\mathrm{C}-\mathrm{C}}$ существенно меньше. Небольшой вклад в $Q_{\text {libr }}$ от барьера внутреннего вращения $Q_{\mathrm{C}-\mathrm{C}}$ не является неожиданным. Оценки этого барьера, основанные на уравнении [24]

$$
Q(\phi)=0.5 Q_{\mathrm{C}-\mathrm{C}}(1-\cos 3 \phi),
$$

показывают, что при $Q_{\mathrm{C}-\mathrm{C}} \approx 12 \mathrm{~kJ} / \mathrm{mol}$ и $\phi=15^{\circ}$ он равняется всего $\sim 2 \mathrm{~kJ} / \mathrm{mol}$, что в большинстве случаев значительно меньше, чем $Q_{\text {libr. }}$ Этим, очевидно, объясняется и примерное совпадение положения пика либрационного поглощения в ИК спектрах полимеров и низкомолекулярных жидкостей, близких по химической структуре к соответствующим мономерным звеньям, например ПС и $\mathrm{C}_{6} \mathrm{H}_{6}$ или поли-4-хлорстирол и $\mathrm{C}_{6} \mathrm{H}_{5} \mathrm{Cl}$ (рис. 2, $a$ и 2,f).

Соотношение $Q_{\mathrm{libr}} \approx E_{\mathrm{coh}} / 3$ оказывается справедливым и для простых жидкостей, а величина $E_{\mathrm{coh}} / 3$ отвечает межмолекулярному потенциальному барьеру смещения молекулярных единиц в конденсированной системе относительно соседей [25]. Этот вид подвижности, впервые охарактеризованный Рейдом и Эвансом [26] как универсальный $\gamma$-процесс в жидкости, является предшественником переориентационного движения молекул, т. е. $\beta$-релаксации. В этом смысле $\beta$-процесс, ближайший к $\alpha$-переходу, является следствием влияния флуктуации непосредственного окружения либрирующей молекулярной единицы, обеспечивающей ее переориентацию при переходе из одной потенциальной ямы в другую. Для полимеров этот релаксационный процесс представляет собой элементарный акт локальной (конформационной) подвижности макромолекулы.

Как видно из табл. 2, потенциальные барьеры для либрации в мономерных звеньях, рассчитанные в настоящей работе с использованием соотношения $v_{\mathrm{libr}}=(c \pi \varphi)^{-1}$ $\left(Q_{\mathrm{libr}} / 2 I\right)^{1 / 2}$, существенно (в 8-11 раз) меньше значений барьеров для элементарных актов сегментального движения $\left(U_{\alpha}\right)$, рассчитанных в работе [11] исходя из температур стеклования $\left(T_{g}\right)$ ПС и его модификаций (табл. 2, столбцы 4,9 и 10). В работе [11] изменения температур стеклования (и, следовательно, изменение барьеров сегментальной подвижности) в случае модификаций бензольного кольца заместителями было связано с изменением массы $M$ (стерические условия подвижности) и с изменениями внутри- и межмолекулярных взаимодействий, которые были охарактеризованы УФ спектрами и дипольными моментами $(\mu)$ модифицированных бензольных колец. Учет взаимосвязи этих факторов (стерического и ,электронного“) позволил объяснить, почему при одинаковой массе образцов 8 и 9 (табл. 1 и 2) у этих полимеров разные температуры стеклования и барьеры для сегментального движения: их мономерные единицы имеют различные дипольные моменты. В то же время образцы 8 и 10 с разной молекулярной массой бензольного кольца имеют одинаковую температуру стеклования, поскольку у них сходные по величине дипольные моменты мономерных единиц (табл. 1, столбец 7).

Однако вполне очевидно, что необходимо учесть и полимерную специфику сегментальной подвижности, заключающуюся в коррелированности движения соседних диполей при фиксированных валентных углах и заторможенности внутреннего вращения в звеньях макромолекулы. Иными словами, локальные условия сегментарного движения (и его потенциальный барьер) определяются не только параметрами мономерных единиц (размер, масса, дипольный момент, оптическая анизотропия и т.д.), но и средним взаимным расположением последних, которое зависит от гибкости макромолекулы [24,27]. То есть если в низкомолекулярных жидкостях подвижностью, отвечающую за $\beta$-релаксацию, является вращательно-диффузионное смещение молекул, то в полимерах это переориентационное движение участка цепи, который является статистически независимым от ориентации соседних участков цепи. Такой статистический сегмент, характеризующий термодинамическую гибкость полимерной цепи, в случае ПС включает 7-8 мономерных звеньев [23], а для замещенных ПС, согласно расчетам [27], он равен 7-11 мономерным единицам. Найденные в литературе $[13,14]$ или рассчитанные по методике [27] значения статистических сегментов $(S)$ изученных в этой работе замещенных ПС приведены в табл. 2, колонка 8.

Зная размер статистического сегмента $(S)$ исследованных полимеров, мы можем оценить высоту барьера для локального сегментарного движения, используя зависимость

$$
U_{\beta}=(0.3 \pm 0.05) E_{\mathrm{coh}}+B,
$$

полученную в [23] для $\beta$-процесса. При относительно небольшом значении параметра $B \approx 10 \pm 5 \mathrm{~kJ} / \mathrm{mol}$, coответствующего барьеру внутреннего вращение вокруг C-С-связи в гибкоцепных полимерах, и используя приближенное равенство $E_{\mathrm{coh}} \approx 3 Q_{\mathrm{libr}}$, мы имеем формулу, по которой эти барьеры вычислялись в настоящей работе:

$$
U_{\beta}=Q_{\mathrm{libr}} S \text {. }
$$

Как видно из табл. 2 (столбцы 10 и 11), рассчитанные на основании полученных данных энергии активации $\beta$-релаксации в ПС и ее модификациях (колонка 11) сопоставимы с барьерами для элементарных актов сегментального движения в тех же замещенных полистиролах, оцененными в работе [11] по их температурам стеклования (колонка 10).

Полученные результаты подтверждают механизм, предложенный в [23] для $\beta$-релаксации, состоящий в поворотном (за счет накопления крутильно-колебательных смещений монозвеньев относительно друг друга) движении корреляционного участка цепи, близкого по величине к статистическому сегменту, с преодолением преимущественно когезионных (межмолекулярных) 
барьеров и при участии однобарьерного транс-гошперехода. Такой акт движения аналогичен акту сегментальной релаксации в расплаве полимера и может реализоваться в твердом полимере в качестве элементарного акта кооперативных конформационных перестроек в $\beta$-процессе. При вырождении кооперативной природы подвижности на высоких частотах $\left(v \geq 10^{7}\right.$ до $\left.10^{8} \mathrm{~Hz}\right)$ оба процесса объединяются в одиночный $\alpha \beta$-процесс с энергией активации $U_{\beta}[28]$.

Выявленная в настоящей работе близость барьеров коррелированного либрационного движения $\left(U_{\beta}\right)$ и барьеров локальной сегментальной подвижности $\left(U_{\alpha}\right)$, определенных по $T_{g}$ методом ДСК, подтверждает, что активационный барьер конформационной подвижности в полимерной цепи мало изменяется в широком интервале температур $T>T_{\beta}$ лишь в силу изменения межцепочечных взаимодействий, оставаясь близким к $U_{\beta}$.

\section{Заключение}

В настоящей работе измерены и проанализированы ИК спектры ПС и его модификаций, полученных путем замещения метильными группами и/или атомами хлора и брома атомов водорода в бензольных кольцах боковых групп макромолекул. Все эти спектры имеют аномально широкую асимметричную полосу поглощения с положением максимума в диапазоне от 40 до $80 \mathrm{~cm}^{-1}$ в зависимости от природы заместителя. Эта полоса поглощения может быть отнесена к поглощению, связанному с либрацией (вращательными колебаниями) фенольных колец, т. е. к поглощению по механизму Поли.

Взаимосвязь между спектральными параметрами поглощения Поли и молекулярными характеристиками исследованных полимеров позволяет непосредственно анализировать роль молекулярной структуры и межмолекулярных взаимодействий в полимерной динамике. Высоты потенциальных барьеров для либрации фенольных колец в макромолекулах ПС и его модификаций $Q_{\text {libr }}$, оцененные на основе анализа этого поглощения, найдены близкими к барьерам активации низкотемпературной механической $\delta$-релаксации в этих полимерах. Сравнение этих барьеров с активационными барьерами локального сегментального движения, определенными методом DSC для тех же ПС, показало, что во всех случаях имеет место равенство $U_{\beta} \approx Q_{\mathrm{libr}} S$ ( $S$ - длина статистического сегмента), подтверждая, что $\beta$-процесс, как элементарный акт сегментального движения в полимерах, связан с корреляционным либрационным движение участка цепи, статистически независящем от соседних участков. В этом смысле универсальный $\delta$-процесс, характеризуемый здесь поглощением по механизму Поли в ИК спектрах, является высокочастотным предшественником (и дополнением) $\beta$-процесса.

В целом результаты, представленные выше, показывают, что терагерцовая ИК спектроскопия позволяет конкретизировать молекулярные механизмы вторичных релаксаций в полимерах и установить взаимосвязь этих процессов с молекулярными характеристиками полимеров, такими как структура мономерной единицы, энергия когезии, потенциальный барьер для внутреннего вращения и термодинамическая жесткость полимерной цепи.

\section{Конфликт интересов}

Автор заявляет, что у него нет конфликта интересов.

\section{Список литературы}

[1] Ngai K.L. Relaxation and Diffusion in Complex Systems. NY: Springer, 2011. $823 \mathrm{p}$.

[2] Готлиб Ю.Я., Тощевиков В.П // Высокомол. соед. 2001. T. 43. № 10. C. 1844.

[3] Parrot E.P.J., Zeitler J.A. // Appl. Spectrosc. 2015. V. 69. N 1. P. 1. doi 10.1366/14-070

[4] Kohler M., Lunkenheimer P., Goncharov Y., When R., Loidl A. // J. Non-Cryst. Solids. 2010. V. 356. N 11. P. 529. doi 10.1016/j.jnoncrysol.2009.07.029

[5] Giraud G., Wynne K. // J. Chem. Phys. 2003. V. 119. N 22. P. 11753. doi $10.1063 / 1.1623747$

[6] Wietzke S., Jansen C., Reuter M., Jung T., Kraft D., Chatterje S., Fischer B.M., Koch M. // J. Mol. Structure. 2011. V. 1006. N 1-2. P. 41. doi 10.16/j.molstruc.2011.0.036

[7] Рыюжов В.А., Берштейн В.А. // ФТТ. 2008. Т. 50. № 10. C. 1901; Ryzhov V.A., Bershtein V.A. // Phys. Solid State. 2008. V. 50. N 10. P. 1985. doi 10.1134/S1063783408100326

[8] Bershtein V.A., Ryzhov V.A. // Adv. Polym. Sci. 1994. V. 114. P. 43. doi $10.1007 / \mathrm{BFb} 0008694$

[9] Hunt N.T., Jaye A., Hellman A., Meech S.R. // J. Phys. Chem. B. 2004. V. 108. N 1. P. 100. doi 10.21/jp035624g

[10] Larkin I. // Trans. Faraday Soc. II. 1973. V. 69. P. 1278. doi 10.1039/F29736901278

[11] Слуцкер А.И., Васильева К.В., Егоров В.М., Докукина А.Ф. // Высокомол. соед. 2002. Т. 44. № 12. С. 2103; Slutsker A.I., Vasil'eva K.V., Egorov V.M., Dokukina A.F. // Polym. Sci. Ser. A. 2002. V. 44. N 12. P. 1255.

[12] Докукина А.Ф., Котон М.М. // Высокомол. соед. 1959. Т. 1. № 8. C. 1129.

[13] Van Crevelen D.W. Properties of Polymers: Their Correlation with Chemical Structure: Their Numerical Estimation and Prediction from Additive Group Combinations. 3rded. Amsterdam and London: Elsevier, 1990. 414 p.; Ван Кревелен Д.В. Свойства и химическое строение полимеров. М: Химия, 1976. 414 с.

[14] Polymer Handbook. $3^{\text {rd }}$ ed. / Ed. by Brandrup J., Immergut E.H. NY:: Wiley-Interscience, 1989. 1904 p.

[15] Krimm S. // Adv. Polym. Sci. 1960. V. 2. P. 51.

[16] Varsanyi G. Vibrational Spectra of Benzene Derivatives. NY: Academic Press, 1969. 43 p.

[17] Jasse B., Monnerie L.J. // Phys. D: Appl. Phys. 1975. V. 8. N 7. P. 863

[18] Kim J.J., McLeish J., Hyde A.J., Bailey R.T. // Chem. Phys. Lett. 1973. V. 22. N 3. P. 503. doi 10.1016/00092614(73)87017-4

[19] Johary G.P. // J. Non-Cryst. Solids. 2002. V. 307-310. N 9. P. 114. doi 10.1016/50022-3093(02)01449-7 
[20] Volkenstein M.V. Conformational Statistics of Polymer Chains. NY. Interscience, 1963. 562 р.; Волькенштейн М.В. Конформационная статистика цепей. М.: Изд. АН СССР, 1959. $466 \mathrm{c}$.

[21] Потапов А.А. // Опт. и спектр. 1996. Т. 81. № 3. С. 420; Potapov A.A. // Opt. Spectrosc. 1996. V. 81. N 3. C. 379.

[22] Coffey W.T., Johary G.P., Kalmykov Ya.P., Titov S.V. // J. Phys. Condens. Matter. 2003. V. 15. N 19. P. 152961. doi 10.1088/0953-8984/15/19/301; Brot $C$, Darmon I. // Molec. Phys. 1971. V. 21. N 5. P. 785. doi 10.1080/00268977100101941

[23] Берштейн В.А., Егоров В.М. Дифференциальная сканирующая калориметрия в физико-химии полимеров. Л.: Химия, 1990. 255 с.; Bershtein V.A., Egorov V.M. Differential Scanning Calorimetry of Polymers. Chichester: Ellis Horwood, 1992. 255 p.

[24] Бирштейн T.М., Птицин О.Б. Конформация макромолекул. М.: Наука, 1984. 391 с.

[25] Глесстон С., Лейдлер К., Эйринг Г. М.Теория абсолютных скоростей реакций. М.: ИЛ, 1948. 584 с.; Glasstone S., Laidler K.J., Eyring $H$. The Theory of Rate Processes. NY: McGraw-Hill Book Co., Inc., 1941. 611 p.

[26] Reid C.J., Evans M.W. // J. Chem. Phys. 1982. V. 76. N 5. P. 2576. doi $10.1063 / 1.443235$

[27] Аскадский А.А., Матвеев Ю.И. Химическое строение и физические свойства полимеров. М.: Химия, 1983. 248 с.

[28] Beiner M., Huth H., Schreder K.J. // Non-Cryst. Solids. 2001. V. 279. N 2. P. 126. doi 10.1016/50022-3093(00)00409-9 